\title{
A Dynamic Foundational Architecture for Semantic Web Services
}

\author{
BRAHIM MEDJAHED \\ Department of Computer and Information Science, University of Michigan, Dearborn \\ ATHMAN BOUGUETTAYA \\ Department of Computer Science, Virginia Tech \\ Recommended by: Ahmed Elmagarmid
}

brahim@umich.edu

athman@vt.edu

\begin{abstract}
The combination of Web services and ontologies is gaining momentum as the potential silver bullets for tomorrow's Web, i.e., the Semantic Web. We propose an architectural foundation for managing semantic Web services in dynamic environments. We introduce the concept of community to cater for an ontological organization of Web services. We develop an ontology called community ontology that serves as a "template" for describing communities of Web services. We also propose a peer-to-peer approach for managing communities in dynamic environments.
\end{abstract}

Keywords: semantic Web, Web services, ontologies, agents

\section{Introduction}

The world has seen a radical shift in economic, social, and political paradigms because of the introduction of the Web. The Web has enabled and created a vast array of opportunities that have transformed and transcended all types of activities. Before the advent of the Web, there was a general "hunger" for data, i.e., data was not readily or cheaply available. What we have witnessed since the Web has emerged, is a complete reversal of that trend where the average user is now literally submerged with too "much" data that is hard to make sense of. While search engines have tried to alleviate this problem, users are still locked into a paradigm that forces them to manually sift through already filtered data. The underlying issue has essentially been finding a strategy to turn Web data into usable information. This is where the Semantic Web comes into play. The Semantic Web aims at augmenting the Web with the necessary tools to allow data to be automatically understood by programs [2]. The building blocks of the envisioned Semantic Web, heavily focused on the use of standards, have already been laid down.

A key issue that has long been overlooked on the Web is the little or lack of broad leveraging of the tremendous investments in Web applications developed over the years to access Web data. These applications are typically customized to access corporate databases. An effective solution to this issue consists of eliciting programmatic access to these applications and viewing them as first class objects so they can be reused (outsourced) and 
combined (composed). Web services are a technology enabler proposed to materialize this vision $[1,10]$. Web services are a recent addition to the Semantic Web to deal with the glut of Web applications. More formally, a Web service is a set of related functionalities that can be programmatically accessed through the Web. Examples of Web services span several application domains that include e-government (e.g., e-tax preparation) and $B 2 B$ e-commerce (e.g., stock trading) $[1,13]$. The maturity of XML-based Web service technologies such as SOAP, UDDI, and WSDL is one of the key factors contributing to the expected wide adoption of Web services [1].

Another concept that is taking the spotlight in the envisioned Semantic Web is the concept of ontology. An ontology is defined as a formal and explicit specification of a shared conceptualization $[2,7,14]$. Ontologies are increasingly seen as key to enabling semanticsdriven data access and processing. They are expected to play a central role to empower Web services with semantics. The combination of these powerful concepts (i.e., Web services and ontologies) has resulted in the emergence of a new generation of Web services called Semantic Web services. Semantic Web services are poised to be the building blocks of tomorrow's Web, i.e., the Semantic Web [10]. Applications "exposed" as Web services would be understood, shared, and invoked by automated tools.

Semantic Web services have spurred an intense activity in industry and academia to address challenging research issues such as the description, selection, monitoring, and composition of Web services. The diversity of these issues calls for the design and development of a Web Service Management System where Web services would be treated as first-class objects that can be manipulated as if they were pieces of data. The research presented in this paper is part of a comprehensive framework we are developing for enabling the automatic composition of Semantic Web services. Web service composition refers to the process of combining several Web services to provide a value-added service, called composite service $[1,10]$. Paramount in this framework is the semantics of Web services. The automatic composition of Web services raises several challenging issues including the meaningful organization and description of Web services, selection of relevant services, composability of participant services (i.e., automatically checking whether they can actually work together), and generating composite service descriptions. Our focus in this paper is on the issue of organizing, describing, and managing semantic Web services.

The semantic organization and description of Web services is an important requirement for deploying the envisioned Semantic Web. The number of Web services available on the Web is increasing fast. Service providers maybe located in different places across the globe. The large scale, dynamics, and heterogeneity of Web services may hinder any attempt for "understanding" their semantics and hence managing them. To address this issue, we propose a Semantic Web centered framework for organizing and describing Web services. We introduce the concept of community to cater for an ontological organization of Web services. Services are clustered into communities based on their domain of interest. We develop an ontology called community ontology that serves as a "template" for describing communities of Web services. A community provides a set of generic operations that can be used "as is" or customized by underlying services. Service providers identify the community of interest and register their service with it. The registration process is handled by a network of software agents associated to service and community providers. An agent is a piece of 
software capable of acting proactively to accomplish tasks on behalf of its consumers (e.g., service providers) [11].

The rest of this paper is organized as follows. In Section 2, we introduce the concept of community to cater for an ontological organization of the service space. In Section 3, we describe operational features of communities via generic operations. In Section 4, we present the technique used for registering Web services with a community. In Section 5, we propose a peer-to-peer approach for managing communities of Web services. In Section 6, we describe our implementation. In Section 7, we give a brief survey of the related work. We finally provide concluding remarks in Section 8.

\section{An ontological framework for semantic Web services}

While the outcomes of our approach are generic enough to be applicable to a wide range of applications, we specifically target the area of government social services providing benefits for senior citizens as a case study. Typically, a large set of services is offered to help senior citizens including FasTran (transportation), Meals on Wheels (meal deliverers), Meals Providers (restaurants), Senior Activity Center (social club for senior citizens). The number of e-government Web services may be large. Some of those services are offered by specialized agencies such as the Department for the Aging. Others are subcontracted from outside organizations including state and federal government agencies (e.g., Department of Health and Human Services), businesses (e.g., restaurants participating in a subsidized government program), volunteer centers (e.g., used by Meals on Wheels service), and nonprofit organizations (e.g., American Red Cross).

\subsection{Ontological support for communities}

Combining the emerging Semantic Web concepts of Web services and ontology are at the core of our approach. To cater for an ontological organization and description of Web services, we propose the concept of community. A community is a "container" that clusters Web services based on a specific area of interest (e.g., disability, adoption). All Web services that belong to a given community share the same area of interest. Communities provide descriptions of desired services (e.g., providing interfaces for insurance services) without referring to any actual service.

We define a metadata ontology, called community ontology, for creating communities of Web services. Metadata ontologies provide concepts that allow the description of other concepts (communities and Web services in our case) [7]. Figure 1 summarizes the proposed model for semantic Web services. Communities are instances of the community ontology. They are created by community providers which use the community ontology as a template. Community providers are generally groups of government agencies, non-profit organizations, and businesses that share a common domain of interest. For example, the Department for the Aging and other related agencies, such as the Department of Health, would define a community that provides healthcare benefits for senior citizens. A community is itself a service that is created, advertised, discovered, and invoked in the same way "regular" Web services are. The providers of a community assign values to the attributes 


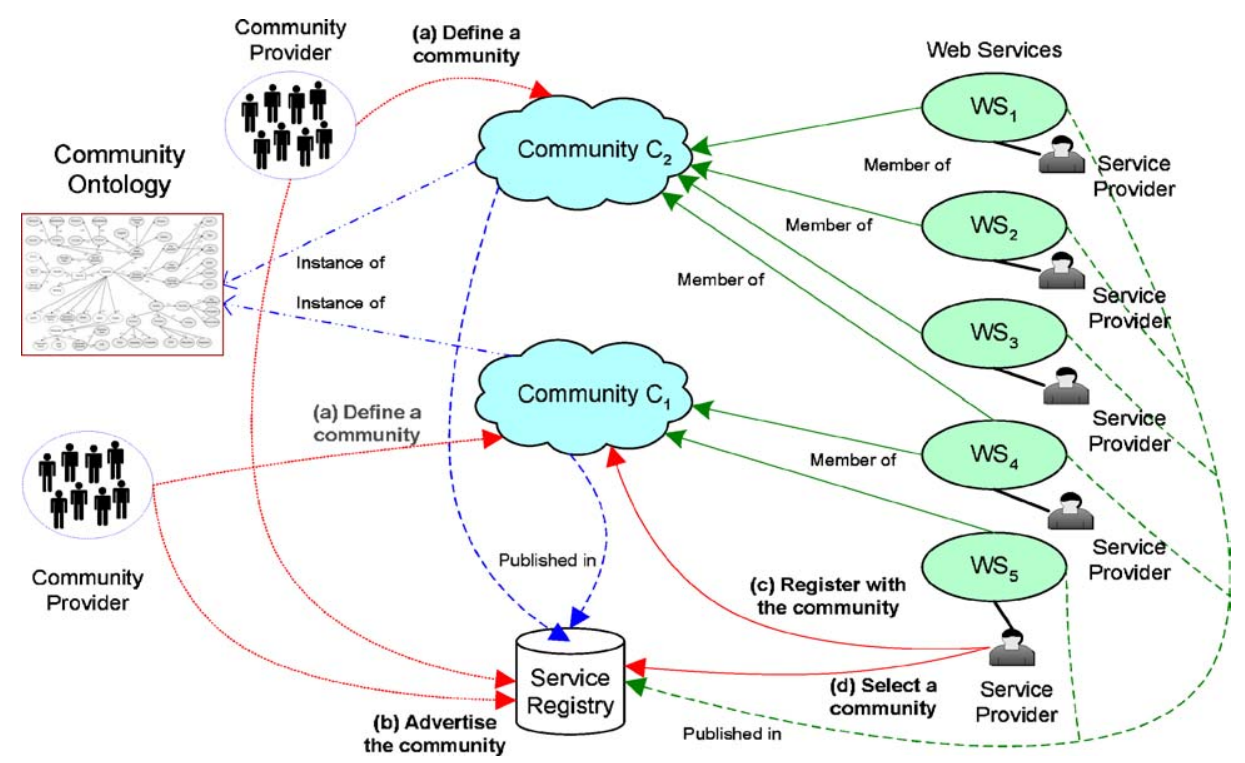

Figure 1. The proposed model for Semantic Web services.

and concepts of the community ontology (figure 1: step a). Communities are published in a registry (e.g., UDDI) so that they can be discovered by service providers (figure 1: step b). Service providers (e.g., medicare provider) identify the community of interest (figure 1: step c) and register their services with it (figure 1: step d). A Web service may belong to different communities. For example a composite service $\left(\mathrm{WS}_{4}\right.$ in figure 1$)$ may outsource operations that have different domains of interest (e.g., "healthcare" and "elderly"). Since these operations belong to two different communities, the composite service is registered with the "healthcare" and "elderly" communities $\left(C_{1}\right.$ and $C_{2}$ in figure 1$)$. End-users (e.g., case officers) select a community of interest and invoke its operations. Each invocation of a community operation is translated into the invocation of a community member's operation.

We use the emerging DAML+OIL language for describing the proposed ontology [9]. DAML+OIL builds on earlier Web ontology standards such as RDF and RDF Schema and extends those languages with richer modeling primitives (e.g., cardinality) [9]. Our approach is not dependent on DAML+OIL; other Web ontology standards (e.g., OWL) could be used to describe the community ontology.

\section{2. $\quad$ Structure of a community}

A community $C_{i}$ is formally defined by a tuple (Identifier ${ }_{i}$, Category $_{i}$, G-operation $_{i}$, Members $_{i}$ ). The identifier ${ }_{i}$ clause contains a unique name and a text description that summarizes $C_{i}$ 's features (figure 2). Category $y_{i}$ describes the area of interest of the community. 


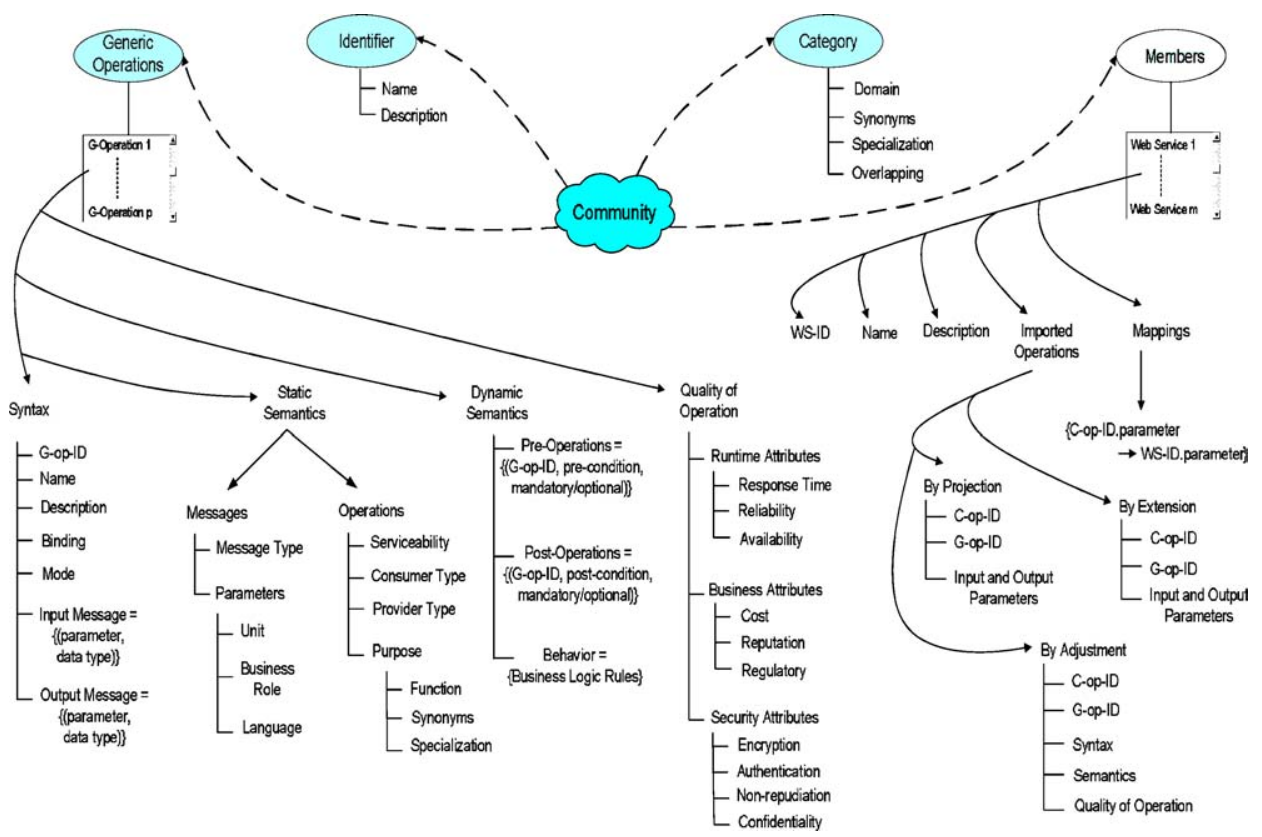

Figure 2. The general structure of a community.

All Web services that belong to $C_{i}$ have the same category as $C_{i}$ 's. $C_{i}$ is accessible via a set of operations called generic operations. Those are specified in the G-operation clause. $_{\text {. }}$. Generic operations are "abstract" operations that summarize the major functions needed by $C_{i}$ 's members. Community providers define generic operations based on their expertise on the corresponding area of interest that is, $C_{i}$ 's category. The term "abstract" means that no implementation is provided for generic operations. Community providers only define an interface for each generic operation $o p_{i k}$. This interface could subsequently be used and implemented by community members (i.e., actual Web services) interested in offering $o p_{i k}$. We say that those members support or import $o p_{i k}$. The execution of $o p_{i k}$ hence refers to the execution of an actual operation offered by a member that support $o p_{i k}$. The Members clause refers to the list of $C_{i}$ 's members. By being members of $C_{i}$, Web service providers "promise" that they will besupporting one or several of $C_{i}$ 's generic operations. In this section, we focus on describing the category clause and community ontology. Details about generic operations and community members are given in Sections 3 and 4 respectively.

The category of a community $C_{i}$ is formally defined by a tuple (Domain ${ }_{i}$, Synonyms $_{i}$, Specialization $_{i}$, Overlapping ${ }_{i}$ ). Domain $i$ gives the area of interest of the community (e.g., "healthcare"). It takes its value from a taxonomy for domain names. For flexibility purposes, different communities may adopt different taxonomies to specify their category. We use XML namespaces to prefix categories with the taxonomy in which they are defined. Simply put, XML namespaces provide a method for qualifying element and attribute names used in XML documents by associating them with URI references. Synonyms $s_{i}$ contains a 
set of alternative domain names for $C_{i}$. For example "medical" is a synonym of "healthcare". Values assigned to this attribute are taken from the same taxonomy as the one used for domains. Specialization ${ }_{i}$ is a set of characteristics of the $C_{i}$ 's domain.For example, "insurance" and "children" are specialization of "healthcare". This means that $C_{i}$ provides health insurance services for children. Communities are generally not independent. They are linked to each other via inter-ontology relationships. These relationships are specified in the Overlapping ${ }_{i}$ attribute. Overlapping ${ }_{i}$ contains the list of categories that overlap with $C_{i}$ 's category. It is used to provide a peer-to-peer topology for connecting communities with

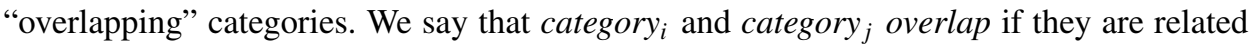
to each other. For example, an operation that belongs to a community whose domain is family may be combined with another operation that belong to a community whose domain is insurance. This would enable providing health insurance for needy families. It should be noted that it is the responsibility of the community providers to identify related categories and assign them to the overlapping attribute.

\subsection{Generic operations}

A generic operation is defined by a set of functional and non-functional attributes. Functional attributes describe syntactic and semantic features of generic operations. Syntactic attributes represent the structure of a generic operation. An example of syntactic attribute is the list of input and output parameters that define the operation's messages. Semantic attributes refer to the meaning of the operation or its messages. We consider two types of semantic attributes: static and dynamic semantic attributes. Static semantic attributes (or simply static attributes) describe non-computational features of generic operations. Those are semantic attributes that are generally independent of the execution of the operation. An example of static attribute is the operation's category. Dynamic semantic attributes (or simply dynamic attributes) describe computational features of generic operations. They generally refer to the way and constraints under which the operation is executed. An example of dynamic attribute is the business logic of the operation i.e., the results returned by the operation given certain parameters and conditions.

Non-functional attributes, also called qualitative attributes, include a set of metrics that measure the quality of the operation. Examples of such attributes include time, availability, and cost. Two services providers that support the same generic operation may have different values for their qualitative attributes. Non-functional attributes model in fact the competitive advantage that Web services may have on each other.

While defining a community, community providers assign values to part of the attributes of their generic operations. The rest of the attributes are assigned either by service providers or third parties during the registration of Web services with $C_{i}$. For example the types of input and output messages (e.g., purchase order, registration confirmation) are define by community providers. The cost (dollar amount) of executing an operation is service-specific and hence defined by the service provider. The other qualitative attributes (e.g., response time, availability) are assigned by third parties (e.g., trusted parties). The way those parties determine the values to be assigned (e.g., via monitoring) is out of the scope of this paper. It is worth noting that the values of some attributes may be assigned by both community and 
service providers. For example, the content of input/output messages is given by community providers. However, service providers may modify this content by adding and/or removing parameters to input and output messages.

\subsection{Community members}

Service providers can, at any time, select a community of interest (based on categories) and register their services with it. The registration process requires giving an identifier (WS-ID), name, and description for the Web service. The identifier takes the form of a unique UUID. The description summarizes the main features of the Web service. Service providers specify the list of generic operations supported by their service through the imported attribute. We define three techniques for importing generic operations: projection, extension, and adjustment. Details about these techniques are given in Section 4. The invocation of an importedoperation is translated into the invocation of an "actual" service operation. The correspondence between imported and "actual" operations is done through the mapping attribute. For each imported operation, the provider gives the ID of the corresponding "actual" operation. It also defines a one-to-one mapping between the imported operation's parameters and "actual" operation's parameters. Defining mappings between parameters enables the support of "legacy" Web services. Providers do not need to change the message parameters in their actual service codes.

Assume that a service provider $S P$ offers a given operation $o p$. The following three cases are then possible: (i) If there is a community $C_{i}$ that contains a generic operation $o p_{i k}$ similar to $o p, S P$ would import $o p_{i k}$ "as is"; (ii) If there is a community $C_{i}$ that contains a generic operation $o p_{i k}$ "closely" similar to $o p$ (e.g., $o p$ has less input parameters than defined in $o p_{i k}$ ), $S P$ would import $o p_{i k}$ using projection, extension, and/or adjustment technique; (iii) If no community has an operation similar or "closely" similar to $o p, S P$ would define a new community $C_{j}$ that has $o p$ as a generic operation and $S P$ 's service as a member. The latter case is similar to the "traditional" WSDL/UDDI/SOAP model where service providers create descriptions for their services. The difference is that, in our case, $S P$ instantiates the attributes and concepts of the community ontology while in the "traditional" model, providers define their service descriptions from scratch.

\section{Operational description of communities via generic operations}

As mentioned previously, a generic operation is described at four different levels: syntactic, static semantic, dynamic semantic and qualitative levels. In this section, we give a detailed description of generic operation attributes for each of those levels.

\subsection{Syntactic attributes}

We identify two levels for syntactically describing a generic operation: operation and message levels. The operation level contains attributes that describe the operation (e.g., name, description of the operation). The message levels caters for the syntactic description 
of messages exchanged by the operation (e.g., name, and data type of a message parameter).

3.1.1. Operation syntax. A generic operation has a unique identifier called $G$-op- $I D$. It takes the form of a Universally Unique ID (UUID). A UUID is an identifier that is unique across both space and time [1]. The operation also has a name and a text description thatsummarizes the operation's features. The binding defines the message formats and protocols used to interact with the operation. An operation may be accessible using several bindings such as SOAP/HTTP, and SOAP/MIME. The binding of an operation is assigned by the service provider. This is in contrast to the rest of syntactic attributes whose values are pre-defined by community providers. Indeed, the binding attribute is dependent on the way the generic operation is implemented at the service provider side. A provider may offer SOAP/HTTP access to a generic operation supported by its Web service while another provider may prefer to use SOAP/MIME for the same operation.

The mode of an operation refers to the order according to which its input and output messages are sent and received. It states whether the operation initiates interactions or simply replies to invocations from other services. We define two operation modes: In/Out or Out/In. One of this values is assigned by community providers to each operation. In/Out operation first receives an input message by a client, process it (locally or forward it to another service), and then returns an output message to the client. Out/In first sends an output message to a server and receives an input message as a result. An example of In/Out operation is checkEligibilityWIC which checks citizen's eligibility for a nutrition program for pregnant women (WIC or Women Infant and Children). As specified in WSDL standard, some operations may be limited to an input or an output message [1]. For example, expirationWIC is an operation that automatically notifies citizens about the termination of their eligibility period for WIC programs. This operation obviously does not require any input message. However, such operations may be considered as In/Out or Out/In operations where the input or output message is empty. Hence, without loss of generality, we focus in the proposed framework on the aforementioned operation modes.

3.1.2. Message syntax. Each input or output message contains one or more parameters. A parameter has a name and data type. We use the set In-Out $(G$-op-ID) to refer to the list of input and output parameters' names. The data type gives the range of values that may be assigned to the parameter. We use XML Schema's built-in data types as the typing system. Built-in types are pre-defined in the XML schema specification. They can be either primitive or derived. Unlike primitive types (e.g., string, decimal), derived types are defined in terms of other types. For example, integer is derived from the decimal primitive type. Although message parameters are predefined by community providers, service providers have the ability to add new parameters, remove some, or change the content (e.g., data type) of pre-defined parameters. In Section 4, we give more details about the way service providers modify the content of operation messages. 


\subsection{Static semantic attributes}

The static semantics of a generic operation refers to a set of attributes whose content is independent of the execution of the operation. It should not only specify the semantics of the operation itself (e.g., what does the operation do) but also the semantics of all messages handled by the operation. In the rest of this section, we define static semantics at two levels: the message parameter and operation levels.

3.2.1. Operation semantics. The static semantic of an operation is defined by the following attributes: serviceability, provider type, consumer type, purpose, and category. Serviceability gives the type of assistance provided by the operation. Examples of values for this attribute are "cash", "in-kind", "informational", and "educational". TANF (Temporary Assistance for Needy Families) is an example of welfare program that provides financial support to needy families. A food stamp is an example of in-kind support available to indigent citizens. Returning the list of senior activity centers is an example of informational support. Enhancing communication skills of visuallyimpaired people is an example of educational support. Other types of support may bementioned by assigning the value "other" to this attribute.

A generic operation may be supported viaone or several provider types. A provider may be governmental ("federal", "state", "local", and "tribal") or non-governmental ("non-profit" and "business") agencies. For example, nursingHome may be provided by the Department of Aging (government agency) and Red Cross (non-profit). The consumer type property specifies the group of citizens (e.g., children, pregnant women) that are eligible to the operation's welfare program. Different groups may be eligible for the same benefit. For example, WIC (Women, Infant, and Children) is a program for pregnant women, lactating mothers, and children.

Each generic operation performs a certain functionality for a specific area of interest. This is specified through the purpose and category attributes respectively. An operation inherits the category of the community in which it is defined. Hence, all operations that belong to the same community share the same category. The purpose attribute describes the goal of the operation. It is defined by three attributes: function, synonyms, and specialization. The function describes the businessfunctionality offered by the operation. Examples of functionsare "eligibility" and "registration". Synonyms and specialization attributes work as they do for categories.

3.2.2. Message semantics. Messages must be semantically described so that they can be "correctly" interpreted by service providers and consumers. We associate a message type $\mathcal{M T}$ to each message. $\mathcal{M T}$ gives a general description of the content represented by the message. For example, a message may represent a purchase order or an invoice. Vertical ontologies are the ideal concept to describe the type of message. Vertical ontologies capture the knowledge valid for a particular domain such as medical, mechanic, chemistry, and electronic [7]. An example of such ontology is RosettaNet's PIPs (Partner Interface Processes) [3].

The message type does not capture the whole semantics of a message. Indeed, it is important to describe the semantics of each parameter belonging to that message. Message 
parameters are so far represented through their name and data type. While data types are an important feature of message parameters, they do not capture their semantics. For example, an eligibility period parameter may be specified in days, weeks, or months. We identify three attributes to model the semantics of message parameters: business role, unit, and language. These attributes are pre-defined by community providers. The business role gives the type of information conveyed by the message parameter. For example, an address parameter may refer to the first (street address and unit number) or second (city and zip code) line of an address. Another example is that of a price parameter. It may represent a total price or price without taxes. Business roles take their values from a predefined taxonomy. Every parameter would have a well-defined meaning according to that taxonomy. An example of such taxonomy is RosettaNet's business dictionary [3]. It contains a common vocabulary that can be used to describe business properties. For example, if the price parameter has an "extendedPrice" role (defined in RosettaNet), then it represents a "total price for a product quantity". For flexibility purposes, different community providers may adopt different taxonomies to specify their parameters' business roles. As for categories, we use XML namespaces to prefix business roles with the taxonomy according to which they are defined.

The unit attribute refers to the measurement units in which the parameter's content is provided. For example, a weight parameter may be expressed in "Kilograms" or "Pounds". A price parameter may be in "US Dollars" or "Euro". We use standard measurement units (length, area, weight, money code, etc.) to assign values to parameters' units. If a parameter does not have a unit (e.g., address), its unit is equal to "none". The content of a message parameter may be specified in different languages. For example, a profession parameter may be expressed in English or Spanish. A translation operation make take as input, an English word (input parameter) and returns as output, its translation in Urdu (output parameter). We adopt the standard taxonomy for languages to specify the value of this attribute. In contrast to the business role whose content is handled only by community providers, the unit and language attributes may changed by service providers. Service providers should have the flexibility to support more units and languages than those specified by community providers. For example, a service provider may decide to support a weight parameter in both "Kilograms" and "Pounds" although the community providers specified "Pounds" as the only measurement unit for this parameter. We give below a formal definition of a message parameter. The definition includes both syntactic and semantic attributes. Each message (input or output) is defined as a set of such parameters.

Definition 1 (Message parameter). A message parameter $P$ is defined as a tuple $(\mathcal{T}, \mathcal{R}$, $\mathcal{U}, \mathcal{L}$ ) where $\mathcal{T}$ is the parameter's data type (in XML Schema), $\mathcal{R}$ is its business role taken from a taxonomy for business roles, $\mathcal{U}$ gives $P$ 's unit of measurement, and $\mathcal{L}$ is the set of languages according to which $P$ may be expressed.

\subsection{Dynamic semantics}

Dynamic semantics allows the description of attributes related to the execution of generic operations. Those attributes may relate the execution of an operation op $\mathrm{p}_{i k}$ to the execution of 
other operations (inter-operation attributes) or describe features inherent to the execution of $\mathrm{op}_{i k}$ (intra-operation attributes). The execution of an operation op ${ }_{i k}$ generally goes through four major observable states: Ready, Start, Active, and end. The execution of $\mathrm{op}_{i k}$ is in the Ready state if the request for executing $\mathrm{op}_{i k}$ has not been made yet. The Start state means that $\mathrm{op}_{i k}$ execution has been initiated. It refers to one of the following events: (i) an input message is sent to $o_{i k}$ if op ${ }_{i k}$ 's mode is $I n / O u t$; or (ii) an output message has been sent from $\mathrm{op}_{i k}$ if $\mathrm{op}_{i k}$ 's mode is $O u t / I n$. We say that $\mathrm{op}_{i k}$ is in the Active state if op $\mathrm{p}_{i k}$ has already been initiated and the corresponding request is being processed. After processing the request, the operation reaches the End state during which results are returned. It refers to one of the following events: (i) an output message is sent to the client if $o_{i k}$ 's mode is $I n / O u t$; or (ii) an input message is received from the server if op ${ }_{i k}$ 's mode is $O u t / I n$. We define a precedence relationship between states, noted $\rightarrow_{t}$, as follows: $S_{1} \rightarrow_{t} S_{2}$ if $S_{1}$ occurs before $S_{2}$.

3.3.1. Pre-operations. Executing a Web service operation may require going through a predefined process that involvesthe execution of several operations called pre-operations.This pre-defined process may be dictated by government regulations. For example, senior citizens must first register with a an Area Agency on Aging via checkRegistration operation before applying for any welfare program. They may also reflect the business logic of the Web service. For example, senior citizens must order a meal from a participating restaurant via the orderMeal operation before requesting its delivery through the mealsOnWheels operation.

Let us consider two generic operations op ${ }_{i k}$ and $\mathrm{op}_{j l}$ that belong to the same or different communities. We say that $\mathrm{op}_{i k}$ is a pre-operation of op $\mathrm{p}_{l}$ if the invocation of $\mathrm{op}_{j l}$ is preceded by the execution of $\mathrm{op}_{i k}$. We call $\mathrm{op}_{i k}$ and $\mathrm{op}_{j l}$ source and target operations respectively. An operation may have several pre-operations. It may also be the source (i.e., pre-operation) of several operations. We give below a formal definition of the pre-operation relationship.

Definition 2 (Pre-operation). Let $\mathrm{op}_{i k}$ and $\mathrm{op}_{j l}$ be two generic operations. $\mathrm{op}_{i k}$ is a preoperation of $\mathrm{op}_{j l}$ if $\operatorname{End}\left(\mathrm{op}_{i k}\right) \rightarrow_{t} \operatorname{Ready}\left(\mathrm{op}_{j l}\right)$.

The definition of a pre-operation relationship includes a source operation $\mathrm{op}_{i k}$, target operation $\mathrm{op}_{j l}$, and the condition and mandatory attributes. The condition is a predicate over op $i k$ 's input and output parameters. op $j l$ can be invoked only if all its pre-operations reached their End state and their conditions are true. If no condition is specified for a given pre-operation then the default value is "true". For example, mealsOnWheels is executed only if the orderMeal operation has been approved. The mandatory attribute takes boolean values and specifies whether the executing the source operation is mandatory or optional. If this attribute is true then the relationship between $\mathrm{op}_{i k}$ and $\mathrm{op}_{j l}$ is obligatory. Otherwise, it is recommended. For example, senior citizens must have ordered meals from a restaurant before requesting home delivery from a volunteer center. However, it is recommended to get the list of participating (participatingRestaurants) restaurants before ordering a meal. Indeed, users can directly order a meal from a restaurant if they already know that restaurant's name. 
3.3.2. Post-operations. The execution of a given operation may trigger the invocation of other operations called post-operations. For example, a pregnant women that registers successfully for a food check program women (registerFoodCheck) is required to register for a nutritional counseling course (registerNutritionCourse). We say that $\mathrm{op}_{i k}$ is a post-operation of $\mathrm{op}_{j l}$ if the termination of op $\mathrm{p}_{j l}$ precedes the invocation of $\mathrm{op}_{i k}$. We call $\mathrm{op}_{j l}$ and $\mathrm{op}_{i k}$ source and target operations respectively. An operation may have several post-operations. It may also be the target (i.e., post-operation) of several operations. Note that if $\mathrm{op}_{i k}$ is a pre-operation of op $\mathrm{p}_{j l}$ then $\mathrm{op}_{j l}$ is not necessarily a post-operation of $\mathrm{op}_{i k}$. For example, checkRegistration is pre-operation of orderMeal. However, orderMeal is not a post-operation of checkRegistration. Indeed, users do not need to order meals whenever their registration with the Department on the Aging is checked. We give below a formal definition of the post-operation relationship.

Definition 3 (Post-operation). Let op ${ }_{i k}$ and op ${ }_{j l}$ be two generic operations. op $\mathrm{p}_{i k}$ is a postoperation of $\mathrm{op}_{j l}$ if $\operatorname{End}\left(\mathrm{op}_{j l}\right) \rightarrow_{t} \operatorname{Ready}\left(\mathrm{op}_{i k}\right)$.

As for pre-conditions, we associate a condition and mandatory attribute to each postoperation relationship. A target operation enters the initiation states if at least one of its source operations has reached its End state and the corresponding condition is true. A postoperation may also bemandatory or optional. For example, a pregnantwomen that registers for a food check program women (registerFoodCheck) must also register for nutritional counseling course by invoking registerNutritionCourse (mandatory $=$ true). The postoperation register is optional (mandatory $=$ false). Indeed, citizens do not necessarily have to register with the Department on the Aging if they are not willing to do so.

3.3.3. Community diagram. Pre and post-operations provide means to specify pre-defined business processes within a community. In the case of e-government, those business processes are mostly driven by government regulations and laws. Several process models have been proposed in the literature such as Petri-nets, statecharts, and $\pi$-calculus. In our approach, we adopt UML activity diagrams as to model pre-operation and post-operation relationships within a community [8]. We refer to such diagram as a community diagram. Activity diagrams are the most widely used process modeling techniques both in conventional interaction technologies (e.g., workflows) and Web services [1]. The reason for their success is their ease-of-use and simplicity for modeling business processes. Several tools (e.g., Rational Rose) are available for designing business processes using activity diagrams. Additionally, the Unified Modeling Language (UML) has become the de facto standard for representing application architecture and design models. Finally, activity diagrams model orchestrations by specifying which actions should be performed, from the beginning of the execution to the end. This seems to be the most natural way in which community and service providers think of a process [1].

Activity diagrams show the flow of activities in a business process. In our approach, each activity represents a generic operation. Generic operation within a diagram may belong to different communities. In this case, the community name prefaces the G-op-IDs. We refer to such pre/post operations as remote pre/post operations. We depict in figure 3 part of the diagramfor the elderly community. The filled circle is the starting point of the 

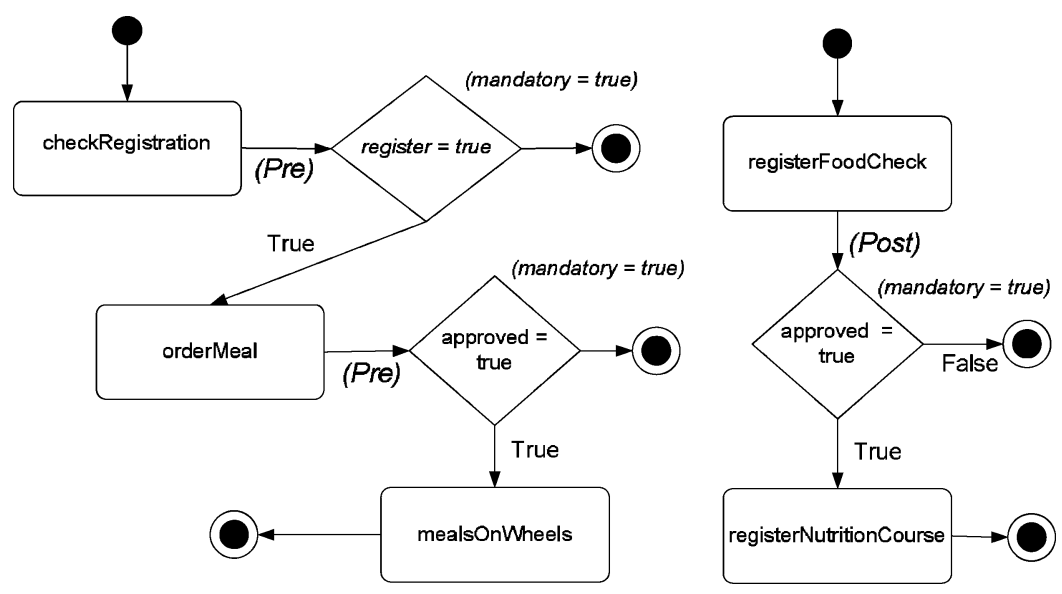

Figure 3. An example of community diagram.

diagram. The filled circle with a border is the ending point. Each edge in the diagram is labeled with a relationship attribute. This attribute takes one of the values "Pre" or "Post" to specify whether the edge models a pre or post-operation. For example, the edge checkRegistration $\rightarrow$ orderMeal models a pre-operation relationship. It specifies that users should execute checkRegistration before initiating the execution of orderMeal. Community diagrams may also indicate that one operation conditionally follows another. For example, the diamond between the checkRegistration and orderMeal operations states that the the value returned by the register parameter should be "true" before invoking orderMeal. The edge registerFoodCheck $\rightarrow$ registerNutritionCourse models a post-operation relationship. It mentions that all citizens that successfully apply for a food check program should register for a nutrition course. Conditional constructs (i.e., diamonds) are labeled with a mandatory attribute defined as in Section 3.3.1. Community diagrams may also model parallelism via fork and join constructs. A fork is represented by a black bar with one flow going into it and several leaving it. It denotes the beginning of parallel processing. A join is depicted by a black bar with several flows entering it and one leaving it. It denotes the end of parallel processing.

3.3.4. Behavior. The behavior of a generic operation op ${ }_{i k}$ refers to the outcome expected after executing op $_{i k}$ given a specific condition. It is defined by a set of business logic rules where each rule $R_{i k}^{m}$ has the following format:

$$
R_{i k}^{m}=\frac{\left(\text { PreParameters }_{i k}^{m}, \text { PreCondition }_{i k}^{m}\right)}{\left(\text { PostParameters }_{i k}^{m}, \text { PostCondition }_{i k}^{m}\right)}
$$

PreParameters ${ }_{i k}^{m}$ and PostParameters ${ }_{i k}^{m}$ are sets of parameters. Each parameter is defined by name, data type, business role, unit, and language as stated in Definition 1 . The elements 
of PreParameters $s_{i k}^{m}$ and PostParameters $s_{i k}^{m}$ generally refer to op ${ }_{i k}$ 's input and output parameters. However, they may in some cases refer to parameters that are neither input nor output of $\mathrm{op}_{i k}$. For example, assume that the address of every citizen registered with the Department on the Aging is stored in the department's database. In this case, this parameter should not be required as input for the orderMeal operation since its value could be retrieved from the database. PreCondition ${ }_{i k}^{m}$ and PostCondition $_{i k}^{m}$ are predicates over the parameters in PreParameters $s_{i k}^{m}$ and PostParameters ${ }_{i k}^{m}$ respectively. The rule $R_{i k}^{m}$ specifies that if PreCondition ${ }_{i k}^{m}$ holds when the operation op $i k$ starts, then PostCondition ${ }_{i k}^{m}$ holds after op ${ }_{i k}$ reaches its End state. If PreCondition ${ }_{i k}^{m}$ does not hold, there are no guarantees about the behavior of the operation. Preconditions generally specify relationships between input values. Similarly, post-conditions generally specify relationships between the returned values. The following is an example of the pre and post condition of a rule associated with the operation registerFoodCheck:

PreCondition: $($ income $<22,090) \wedge($ familySize $\geq 2) \wedge($ zipCode $=22044)$
PostCondition: $($ approved $=$ true $) \wedge($ duration $=6)$

The rule uses the parameters income (unit $=\{$ year, US dollar $\}$ ), familySize, and zipcode in the pre-condition. The attributes approved and duration (unit $=\{$ month $\}$ ) are used in the post-condition. The rule specifies that citizens with a yearly income less than 22,090 US dollars and a minimum household size 2 are eligiblefor food checks and the eligibility period is 6 months.

\subsection{Qualitative properties}

Multiple Web services that belong to the same community may import the same generic operation. It is hence important to define a set attributes that help select the "best" Web service supporting a given functionality. For this purpose, we define a Quality of Operation (QoP) model based on a set of qualitative attributes that are transversal to all operations such as the cost and response time.

The international quality standard ISO 8402 describes quality as "the totality of features and characteristics of a product or service that bear on its ability to satisfy stated or implied needs" $[12,15]$. Wedefine QoP as a set of non-functional attributes that may impact the quality of the operations imported by a Web service. There are many QoP attributes important to Web services operations. We organize them into three groups of quantifiable attributes based on type of measurement performed by each attribute: run-time, business, and security. Note that other attributes and groups may be added to our QoP model without altering the generality of our approach.

Run-time attributes. These attributes enable the measurement of properties that are related to the execution of an operation ${ }_{i} p_{i k}$. We identify three run-time attributes: response time, reliability, and availability. The response time measures the expected delay in seconds between the moment when $o p_{i k}$ ) enters the Start state (i.e., $o p_{i k}$ is initiated) and reaches 
the End state (i.e., $o p_{i k}$ gets or sends the results). Time $\left(o p_{i k}\right)$ is computed using the expres-

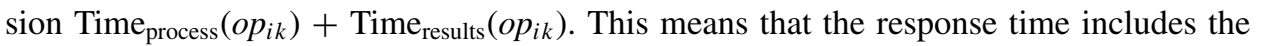
time to process the operation (Time process ) and the time to transmit or receive the results (Time results $_{\text {. }}$. The reliability of $o p_{i k}$ is the ability of the operation to be executed within the maximum expected time frame. Reliability $\left(o p_{i k}\right)$ is computed based on historical data about previous invocations of the operation using the expression $\mathrm{N}_{\text {success }}\left(o p_{i k}\right) / \mathrm{N}_{\text {invoked }}\left(o p_{i k}\right)$ where $\mathrm{N}_{\text {success }}\left(o p_{i k}\right)$ is the number of times that the operation has been successfully executed within maximum expected time frame and $\mathrm{N}_{\text {invoked }}\left(o p_{i k}\right)$ is the total number of invocations. The availability is the probability that the operation is accessible. Availability $\left(o p_{i k}\right)$ is measured by the expression UpTime $\left(o p_{i k}\right) / \operatorname{TotalTime}\left(o p_{i k}\right)$ where UpTime is the time $o p_{i k}$ was accessible during the total measurement time TotalTime.

Business attributes. These attributes allow the assessment of an operation $o p_{i k}$ froma business perspective. We identify three business attributes: cost, reputation, and regulatory. The cost gives the dollar amount required to execute $o p_{i k}$. The reputation of $o p_{i k}$ is a measure of the operation's trustworthiness. It mainly dependson users' experiences on invoking $o p_{i k}$. Users are givena range to rank Web service operations (e.g., between 1 and 10). The lowest value refer to the best ranking. Different users may have different opinions on the same operation. The reputation of $o p_{i k}$ is defined by the average ranking given by users to the operation. Reputation $\left(o p_{i k}\right)$ is computed by the expression $\sum_{u=1}^{n} \operatorname{Ranking}_{u}\left(\operatorname{op}_{i k}\right) / n$, where Ranking R $_{u}$ is the ranking by user $u$ and $\mathrm{n}$ is the number of the times the operation has been ranked. The regulatory property is a measure of how well $o p_{i k}$ is aligned with government regulations. Regulatory $\left(o p_{i k}\right)$ is value within a range (e.g., between 1 and 10). The lowest value refer to an operation that is highly compliant with government regulations.

Security attributes. These attributes describe whether the operation $o p_{i k}$ is compliant with security requirements. Indeed, service providers collect, store, process, and share information about millions of users who have different preferences regarding security of their information. We identify four properties related to security and privacy: encryption, authentication, non-repudiation, and confidentiality. Encryption is a boolean that indicates whether $o p_{i k}$ 's message aresecurely exchanged (using encryption techniques) between servers and clients. Authenticationis a boolean that states whether ${ } p_{i k}$ 's consumers (users and other services) are authenticated (e.g., through passwords). Non-repudiation is a boolean that specifies whether participants (consumers and providers) can deny requesting or delivering the service after the fact. The confidentiality attribute indicates which parties are authorized to access the operation's input and output parameters. Confidentiality $\left(o p_{i k}\right)$ contains $o p_{i k}$ 's input and output parameters that should not be divulged to external entities (i.e., other than the service provider). If a parameterdoes not belong to Confidentiality $\left(o p_{i k}\right)$, then no confidentiality constraint is specified on that parameter. Assume that confidentiality $\left(\mathrm{op}_{i k}=\{\mathrm{SSN}\right.$, salary $\}$ where SSN, salary are two $o p_{i k}$ 's input parameters. The content of this attribute states that those two parameters are kept private by $o p_{i k}$ 's provider. 


\section{Registering Web services with communities}

Registering A Web service with a community refers to the process of importing generic operations. The invocation of an imported operation is translated into the invocation of an "actual" service operation. The correspondence between imported and "actual" operations is done through the mapping attribute. For each imported operation, the provider gives the ID of the corresponding "actual" operation. It also defines a one-to-one mapping between the imported operation's parameters and "actual" operation's parameters. Defining mappings between parameters enables the support of "legacy" Web services. Providers do not need to modify the message parameters in their actual service codes.

\subsection{The Web service registration process}

The registration process is handled by a network of software agents associated to service and community providers. Member and community agents $\left(M A_{j}\right.$ and $\left.C A_{i}\right)$ are attached to each service and community provider $\left(S P_{j}\right.$ and $\left.C P_{i}\right)$ respectively (figure 4$) . M A_{j}$ handles the registration of $S P_{j}$ 's Web services with the community $C_{i} . S P_{j}$ registers its service $W S-I D$ using the following registration statement:

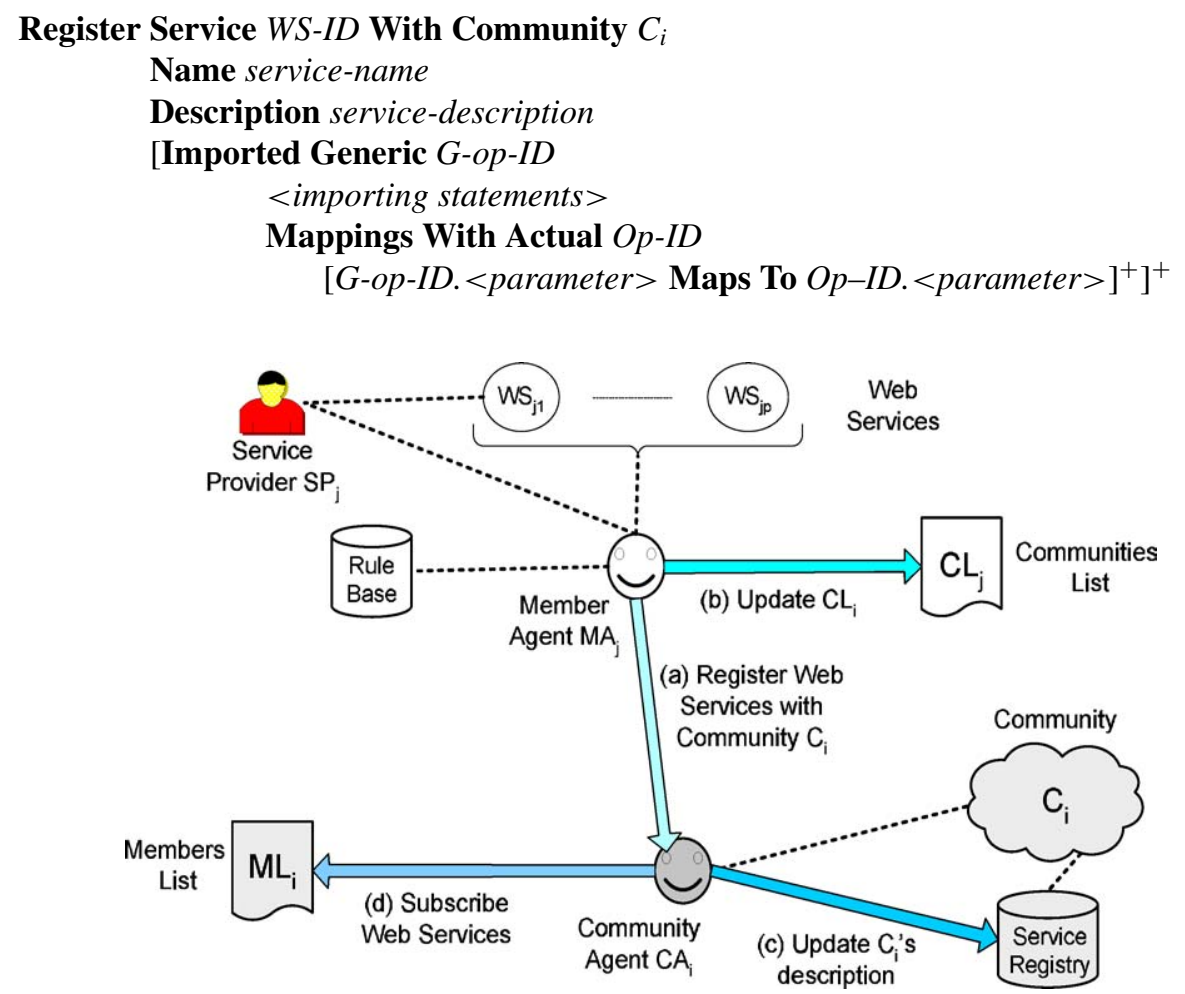

Figure 4. The web service registration process. 
The clauses in the aforementioned statement correspond to the different attributes defined for service members within the community ontology. The $<$ importing statements $>$ is a sequence of statements for importing generic operation. A Web service may import several generic operations as stated by the "+" iteration symbol.

$M A_{j}$ parses the registration statement and sends a registration message $S P \_$Register (WS$I D$,name,desc,imported) to $C A_{i}$ (figure 4, step (a)). The message includes the service ID (WS-ID), name, description, and the list of imported operations. The imported set is equal to $\{(G$-op-ID,mappings,AttSet,NotImpSet $)\}$. It includes the mappings of G-op-ID (ID of the imported operation) with actual service operation. AttSet is the set of G-op-ID's attributes with their values as assigned by $S P_{j}$. It is defined by the set $\{$ (attribute,value $\left.)\right\}$. NotImpSet is the list of non-imported attributes. $M A_{j}$ maintains a Communities List $C L_{j}$ and Rule Base $R B_{j} . C L_{j}$ is the list of communities with which $S P_{j}$ 's Web services are registered. Each entry in this list contains the G-op-ID of an imported operation, the WS-ID of the service that imported it, and the ID (CA-ID) of the community agent $C A_{i}$. To enable fast access to $C L_{j}$, we sort it on the G-op-ID column using Counting Sort algorithm [6]. $R B_{j}$ contains a set of rules that enable $M A_{j}$ to react to changes issued by community providers.

Upon reception of the registration message, $C A_{i}$ updates the content of $C_{i}$ 's members list $M L_{i}$ (figure 4, steps (c) and (d)). Each entry in $M L_{i}$ contains the ID of the imported operation (op-ID), the ID of the importing service (WS-ID), the ID of WS-ID's agent (MA$I D)$, WS-ID's status ("available ", "unavailable", or "unsubscribed"), and the list NotImp of G-op-ID's attributes not imported by WS-ID. The NotImp column is assigned with the content of NotImpSet included in the registration message. We sort $M L_{i}$ on the op-ID column using Counting Sort algorithm to enable fast access to $M L_{i}[6]$.

Figure 5 gives the algorithm executed by a community agent $C A_{i}$ and member agent $M A$ during registration. SP_Register(WS-ID,name,desc,imported) describes the actions executed by $\mathrm{CA}_{i}$ as a reaction to a registration message sent by $M A$. The Insert_Member() function allows the insertion of a new member in $\mathrm{C}_{i}$ 's description. Insert_ML() function allows the insertion of a new entry in the $\mathrm{ML}_{i}$ list for members.

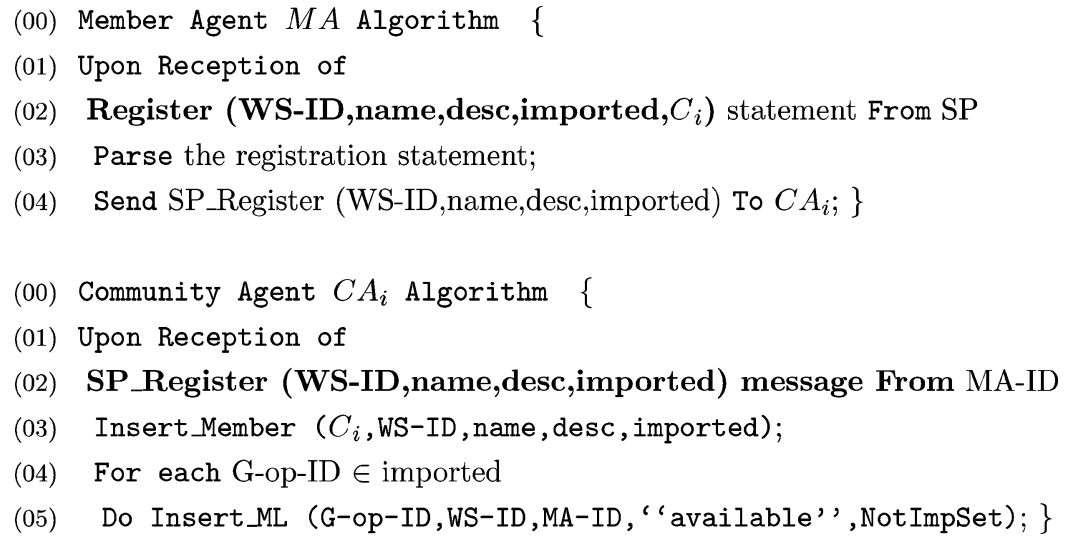

Figure 5. Service registration: Member and community agent algorithms. 


\subsection{Importing generic operations}

Service providers use generic operations as "templates" to define their operations. A Web service may offer all or some of the generic operations defined within a community. The provider specifies the G-op-IDs of the operations imported by its service. By adopting a generic operation, the service provider "promise" to abide by all attributes (syntactic, semantic, and behavioral) of that operation except those changed explicitly during importation. Providers may customize generic operations to best fit their capabilities via importing statements. Customization has the important advantage of enabling flexible and personalized Web service descriptions. It is important to note that customization process does not affect the description of generic operations. Two service providers may import the same generic operation in different ways.

We define three importing statements: projection, extension, adjustment. Importing statements are defined within member agents. Projection, extension, and adjustment may be combined to define imported operations. For example, a service provider may use projection and extension to remove existing parameters from a generic operation and add new ones.

Projection. A generic operation G-op-ID imported by projection uses a subset of the input/output parameters defined in G-op-ID. The rest of message parameters are not imported by the service and hence, are included in the NotImpSet sent by the member agent to the community agent. Assume that a checkRegistrationAAA operation includes register (boolean) and registrationDate (date) as output parameters. A service provider may customize this operation by keeping only the register parameter if it is not interested in returning citizen's registration date. In what follows, we give the general form of a Project statement. The input and output clauses give the subset of G-op-ID's parameters supported by the imported operation:

\section{Project $G$-op-ID \\ Input $<$ list-of-parameter-names $>$ \\ Output $<$ list-of-parameter-names $>$}

The AttSet submitted with the registration message takes the form $\{($ att,value $)\}$ where att is a projected parameter and the content of value is "null" since no new value is assigned to the input or output parameter. We refer to AttSet.Attributes as the list of projected parameters. The list of not imported attributes NotImpSet is defined by the expression $\left(\operatorname{In}\left(o p_{i k}\right) \cap\right.$ Out $\left(\right.$ op $\left.\left._{i k}\right)\right)$ - AttSet.Attributes.

Extension. An imported operation defined by extension adds input and/or output parameters to the corresponding generic operation. The new parameters and their values are included in the registration messages sent by the member agent to the community agent. For example, a service provider (e.g., a volunteer center) may extend the mealsOnWheels operation by adding the deliverer's cell phone number as an output parameter. Service providers must assign values to the attributes of each new message parameters, namely data type, unit, business role, and language. Below is the general form of an extension statement: 


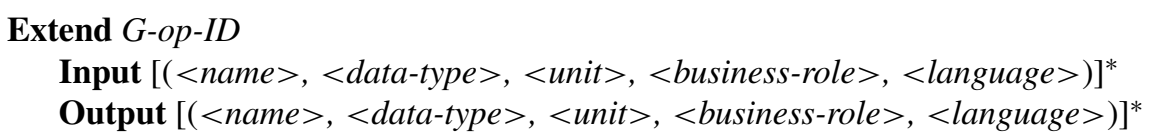

The AttSet submitted with the registration message takes the form $\{($ att,value $)\}$ where att is a new attribute added by the extension statement and value $=(<$ name $>,<$ data-type $>$, $<$ unit $>,<$ business-role $>,<$ language $>$ ). The list of not imported attributes NotImpSet is empty since all message parameters are imported.

Adjustment. The aim of an adjustment statement is to modify the content of a generic operation's attributes. Service providers may assign values to attributes whose content is undefined (e.g., qualitative attributes) or change the content of previously assigned attributes (e.g., language attribute). Adjustment is done by adding a value to an attribute (Add clause) or deleting an existing value from it (Delete clause). For example, service providers may modify the language attribute if their operation supports a language different from the one specified by community providers. The Add and Delete clauses may be combined to remove and add values to an attributes. The new and deleted values are included in the registration messages sent by the member agent to the community agent. The general form of an adjustment statement is given below:

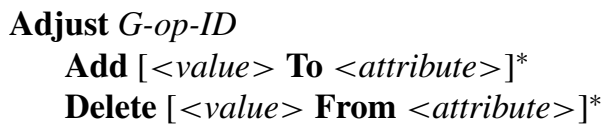

The AttSet submitted with the registration message takes the form $\{$ (att,[“+"/“-”],value) $\}$ where att is an attribute modified by the adjustment statement. The value is preceded by a "+" or "-" symbol depending on whether that value is added or deleted from att respectively. The list of not imported attributes NotImpSet is empty since all message parameters are imported.

\section{A peer-to-peer approach for managing communities}

Communities and their members operate in a highly dynamic environment where changes can be launched to adapt to actual business climate (e.g., economic, politic, organizational). Changes are initiated by community or service providers. At the community providers side, generic operations may be dynamically added, deleted, and modified. If a generic operation G-op-ID is deleted or modified, then all members that are supporting G-op-ID should be notified to ensure global consistency. At the service provider side, a Web service may cancel its membership with a community, make its operations temporarily unavailable, or modify the definition of its imported operations. The community provider should in this case be notified to avoid references to inexistent or obsolete imported operations.

In our approach, all changes are introduced through member and community agents. Agents automatically interact with their peers to manage changes. We consider two types of changes based on the party that launched them: community or service providers. 


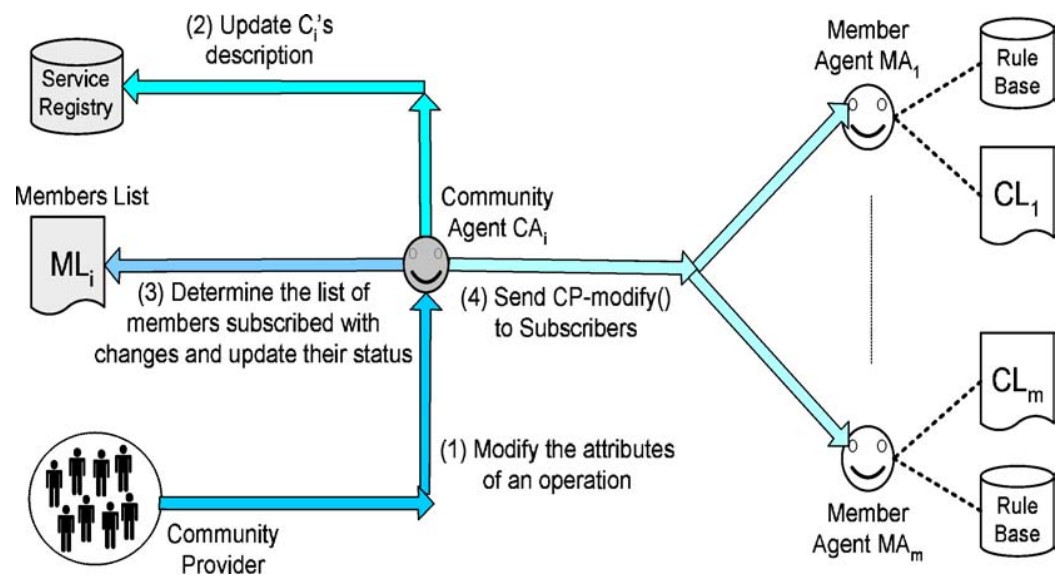

Figure 6. Propagating changes initiated by a community provider to its members.

\subsection{Propagating changes initiated by community providers}

Community providers (CPs) may modify the definition of their generic operations (figure 6). For example, they may change the pre-operations attribute to reflect new government regulations. For this purpose, each $\mathrm{CP}_{i}$ executes a Modify statement defined in its $\mathrm{CA}_{i}$ agent (figure 6, step 1). The statement includes the G-op-ID of the operation to be modified by $\mathrm{CP}_{i}$ and a ModifySet that contains the list of attributes to be modified along with their new content. ModifySet is defined by the set $\{(<$ attribute $>,["+" /$ "_-"], $<$ value $>)\}$. We use the notation ModifySet. Att to refer to the set of modified attributes. $\mathrm{CA}_{i}$ will then access the service registry and update $\mathrm{C}_{i}$ 's description by changing the content of ModifySet.Att's attributes (figure 6, step 2). In the third step, $\mathrm{CA}_{i}$ accesses $\mathrm{ML}_{i}$ list to determine the list $\mathcal{L}$ of members subscribed with $\mathrm{CP}_{i}$ 's changes. A member WS-ID is subscribed with $\mathrm{CP}_{i}$ 's changes if WS-ID imports G-op-ID and at least one attribute in ModifySet.Att is imported by WS-ID. $\mathrm{CA}_{i}$ assigns the value "unavailable" to the status of each subscribed member. This prevents references to members that imported "obsolete" generic operations (figure 6, step 3). Finally, $\mathrm{CA}_{i}$ sends a CP-Modify(G-op-ID,WS, ModifySet) notification to each subscribed member's $\mathrm{MA}_{j}$ (figure 6, step 4). We give below a formal definition of a subscription:

Definition 4 (Subscription). Let $\mathrm{C}_{i}$ be a community and $W S_{j}$ be a member that imported an operation op ${ }_{i k} . W S_{j}$ is subscribed with changes specified in CP-Modify(G-op-ID,ModifySet) if (i) G-op-ID $=\mathrm{op}_{i k}$ and (ii) ModifySet.Att $-M L_{i}[k]$. NotImp $\neq \emptyset$ where $k$ is the entry corresponding to G-op-ID and $\mathrm{WS}_{j}$.

Because pre and post-operations for a given operation ${ } p_{i k}$ may belong to different communities, we associate two "dual" subscription lists $\mathrm{PPCS}_{i}$ and $\mathrm{PPMS}_{i}$ to each community agent $C A_{i}$. These lists are used to notify relevant parties (communities and members) about 


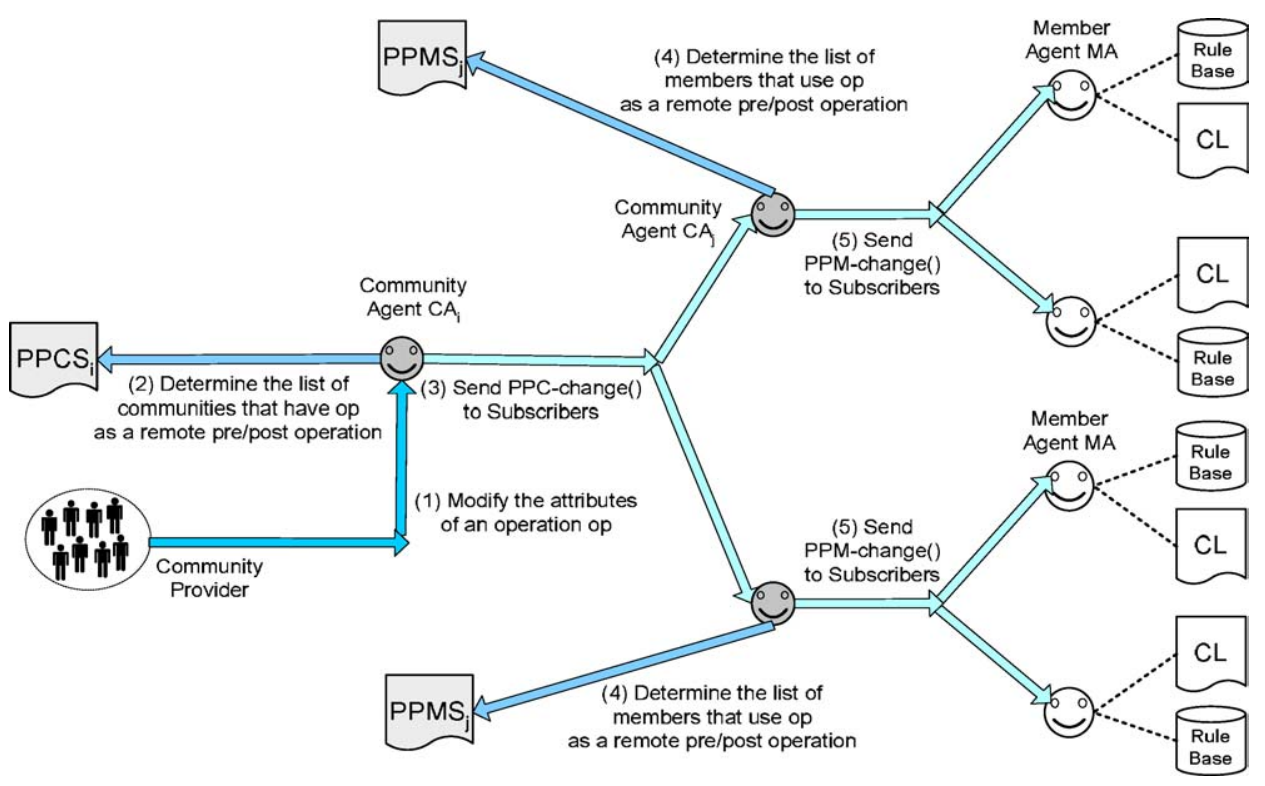

Figure 7. Propagating changes initiated by community providers to their peers.

changes that are related to remote pre and post-operations. PPCS $_{i}$ (Pre and Post-operation Community Subscription) contains the list of communities $C_{j}$ that use an operation ${ } p_{i k}$ of $C_{i}$ as a remote pre or post-operation. Each entry in $\mathrm{PPCS}_{i}$ contains the ID (op-ID) of $o p_{i k}$ and the ID of $C_{j}$ 's agent (CA-ID). Such entry is created at $C_{j}$ 's definition time; at that time, $C A_{j}$ sends a $C P-P P$-subscribe-CP(op-ID) to $C A_{i}$ (CP stands for Community Provider and $\mathrm{PP}$ for Pre/Post operation). $\mathrm{PPCS}_{i}$ is used to notify $C_{j}$ about changes that occur in $o p_{i k}$. Each time $\mathrm{CP}_{i}$ executes a Modify statement on $o p_{i k}, C A_{i}$ accesses $\mathrm{PPCS}_{i}$ list and sends a notification $C P$-PP-Alert-CP $\left(\right.$ op $\left._{i k}\right)$ to each community agent $C A_{j}$ that uses op ${ }_{i k}$ as a remote pre or post-operation (figure 7). We sort $P P C S_{i}$ on the op-ID column using Counting Sort algorithm to enable fast access to the list [6]. Once the CP-PP-Alert-CP(op $\left.{ }_{i k}\right)$ message is received by $C A_{j}, C A_{j}$ notifies $C_{j}$ 's members using the $\mathrm{PPMS}_{j}$ list.

$\mathrm{PPMS}_{j}$ (Pre and Post-operation Member Subscription) contains the list of $C_{j}$ 's members that use the operations $o p_{i k}$ of other communities $C_{i}$ as remote pre or post-operations. This list is created at $C_{j}$ 's definition time. It is used to notify members about changes that occur in their pre and post-operations. Each entry in $\mathrm{PPMS}_{j}$ contains the ID (CA-ID) of the community agent $C A_{i}$, the ID (PP-ID) of the remote pre or post-operation $o p_{i k}$, and the ID (MA-ID) of the agent of a $C_{j}$ 's member that uses $o p_{i k}$ as a remote pre or post-operation. $C A_{j}$ accesses the PPMS ${ }_{j}$ list and sends a notification message CP-PP-Alert-SP $\left(o_{i k}\right)$ (SP stands for Service Provider) to each member agent MA-ID that uses $o p_{i k}$ as a remote pre-operation (figure 7). We sort $P P M S_{i}$ on the CA-ID column using Counting Sort algorithm to enable fast access to the list [6]. To prevent references to members that imported "obsolete" generic operations, $\mathrm{CA}_{j}$ assigns the value "unavailable" to the status of MA-ID entries in $M L_{j}$ list. 


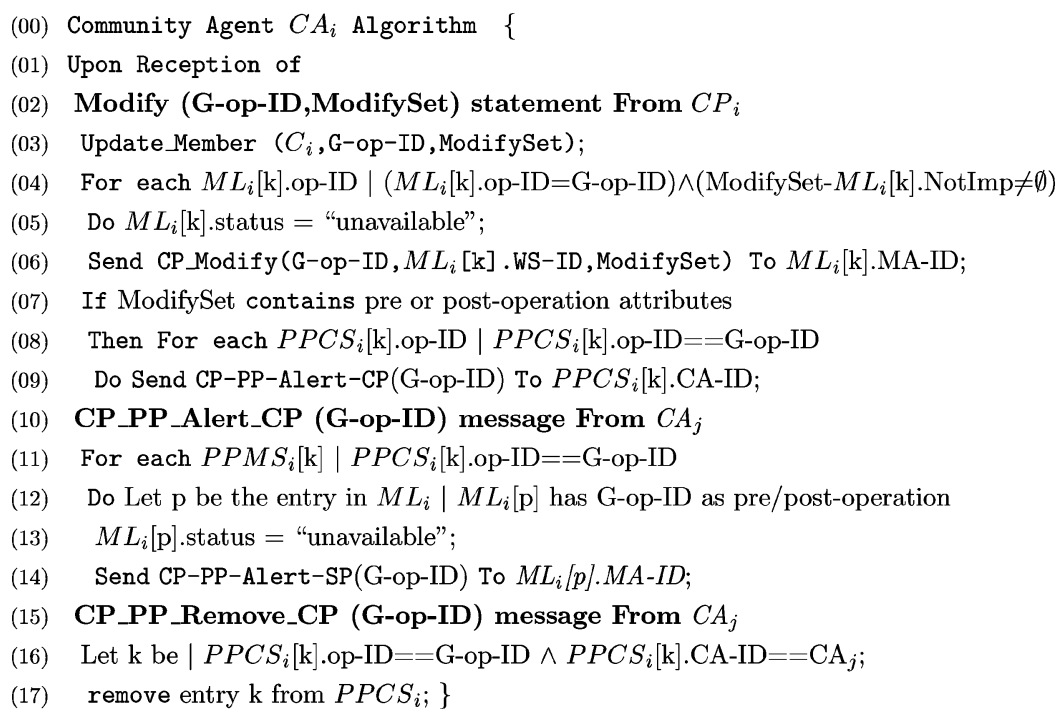

Figure 8. Generic operation modification: Community agent algorithm.

We only consider entries in which MA-ID imported operations that have $o p_{i k}$ as a remote pre or post-operation.

$\mathrm{C}_{j}$ 's provider may remove the remote pre/post operation $o p_{i k}$ from its generic operation definitions. To propagate this change, $\mathrm{CA}_{j}$ removes from $\mathrm{PPMS}_{j}$ the entry that corresponds to $C A_{i}$ and $o p_{i k}$. $\mathrm{CA}_{j}$ also sends a $C P$-PP-Remove-CP(op $\left.{ }_{i k}\right)$ to $C A_{i}$. Upon reception of this message, $C A_{i}$ removes from $\mathrm{PPCS}_{i}$ the entry corresponding to $C A_{j}$ and $o p_{i k} . C A_{i}$ will hence send no $C P$-PP-Alert-CP(op $\left.i k\right)$ messages to $C A_{j}$ since $\mathrm{C}_{j}$ does not use $\mathrm{op}_{i k}$ as a remote pre or post operation. Figure 8 summarizes the algorithm executed by $C A_{i}$ for managing the modification of generic operations. The Update Member() function allows the update of the description of a community description within the service registry.

\subsection{Propagating changes initiated by service providers}

The provider of a Web service WS-ID may initiate changes that should be sent to their communities (figure 9). A community $C_{i}$ is notified about a change if the change is made on an operation imported from $C_{i}$. All changes are introduced through member agents and automatically forwarded to community agents which reflect those changes at the community level. We define the following service providers changes:

- Modifying operations. WS-ID's provider may modify attributes (e.g., remove a message parameter) of a previously imported operation through modification statements defined in WS-ID's agent $M A$ (figure 9, step 1). A modification statement includes the $G$-op-ID 

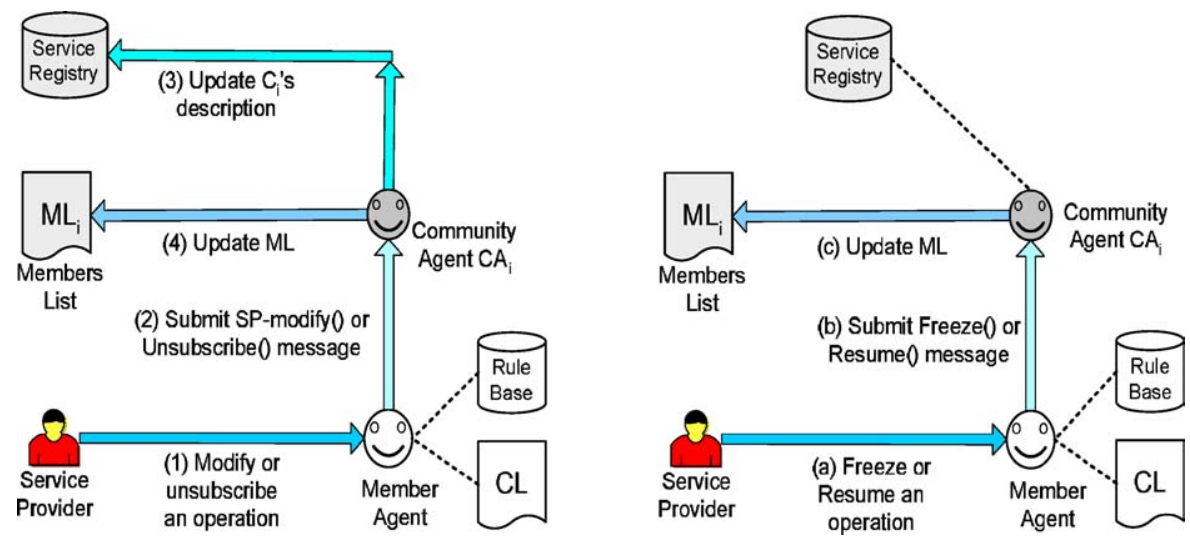

Figure 9. Propagating changes initiated by service providers.

of the operation to be modified, WS-ID of the service that imported it, and the importing statement I-statement used to do the modification. Once $M A$ gets a modification statement, it sends an SP-Modify(G-op-ID,WS-ID,IType,ModifySet) message to $C_{i}$ 's agent $C A_{i}$ (figure 9, step 2). ModifySet is defined as in CP-Modify() messages. IType gives the type of I-statement ("projection", "extension", "adjust add", and "adjust delete"). Upon reception of the $S P$-Modify() message, $C A_{i}$ updates $C_{i}$ 's description (figure 9, step 3). If the message concerns a projection statement, $C A_{i}$ updates the NotImp column in the $\mathrm{ML}_{i}$ 's entry that correspond to G-op-ID and WS-ID (figure 9, step 4). It assigns the content of ModifySet.Att to this column. If the message concerns an "adjust delete" statement on a remote pre or post operation $o p_{j l}$, then $C A_{i}$ removes the entry in $\mathrm{PPMS}_{i}$ corresponding to $C A_{j}, o p_{j l}$, and WS-ID's agent. If the message concerns an "adjust add" statement on a remote pre or post operation $o p_{j l}$, then $C A_{i}$ adds a new entry in $\mathrm{PPMS}_{i}$ with the values $C A_{j}, o p_{j l}$, and WS-ID's agent $M A-I D$.

- Freezing operations. An imported operation may be "available", "unavailable" (e.g., due to network problem), or "unsubscribed". WS-ID's provider may temporarily make an imported operation G-op-ID operation by executing the Freeze statement (figure 9, step a) defined in $M A$. The statement includes the G-op-ID of the operation to be frozen and the WS-ID of the service that imported it. As a consequence, MA sends an SP-Freeze(G-op$I D, W S-I D$ ) message to $C A_{i}$ (figure 9, step b). $C A_{i}$ then assigns the "unavailable" value to the status column of the $\mathrm{ML}_{i}$ 's entry that correspond to G-op-ID and WS-ID (figure 9, step c).

- Resuming operations. WS-ID's provider may re-activate an operation G-op-ID that has previously been frozen through the Resume statement defined in $n M A$. The statement includes a reference to WS-ID and G-op-ID. As a consequence, $M A$ sends an $S P$-Freeze $(G$ $o p-I D, W S-I D$ ) to G-op-ID's community agent $C A_{i}$ (figure 9, step b). $C A_{i}$ then assigns the "available" value to the status column of the $\mathrm{ML}_{i}$ 's entry that correspond to G-op-ID and WS-ID (figure 9, step c). 
- Unsubscribing operations. WS-ID's provider may decide not to support an imported operation any more. For that purpose, it executes MA's Unsubscribe statement (figure 9, step 1). $M A$ informs $C A_{i}$ about this change (figure 9, step 2) by sending an Unsubscribe $(G$ $o p-I D, W S-I D$ ) message to it. $C A_{i}$ then updates $C_{i}$ 's description (figure 9, step 3). It also assigns the "unsubscribed" value to the status of the $\mathrm{ML}_{i}$ 's entry that correspond to $G$ $o p-I D$ and WS-ID (figure 9, step 4). $C A_{i}$ periodically checks the status column in $\mathrm{ML}_{i}$ and remove all entries associated with unsubscribed operations.

Figures 10 and 11 depict the algorithm executed by community and member agents for managing changes initiated by service providers. The Lookup_Community() function (lines 14) is executed on the communities list $C L$. It returns the $C A_{i}$ 's ID of the community $C_{i}$ to which the operation G-op-ID belongs.

Each member's agent $\mathrm{MA}_{j}$ also needs to react to change notifications sent by a community agent via CP-Modify(G-op-ID, WS ${ }_{j}$, ModifySet) or CP-PP-Alert-SP $\left(\right.$ op $\left._{i k}\right)$ messages. The actions to be performed by $\mathrm{MA}_{j}$ as a result of change notification are captured using ECA (Event Condition Action) rules [5]. Briefly, the basic semantics of an ECA rule is as follows: when an event occurs, an action is executed if the corresponding condition is true. Event-driven systems are becoming the paradigm of choice for organizing many classes of loosely coupled and dynamic applications. Members react to changes using their own change control policies via local rule specified in their agent. Hence, the reaction to changes can be customized to the peculiarities of each member. Below is an example of ECA rule specified within a member agent:

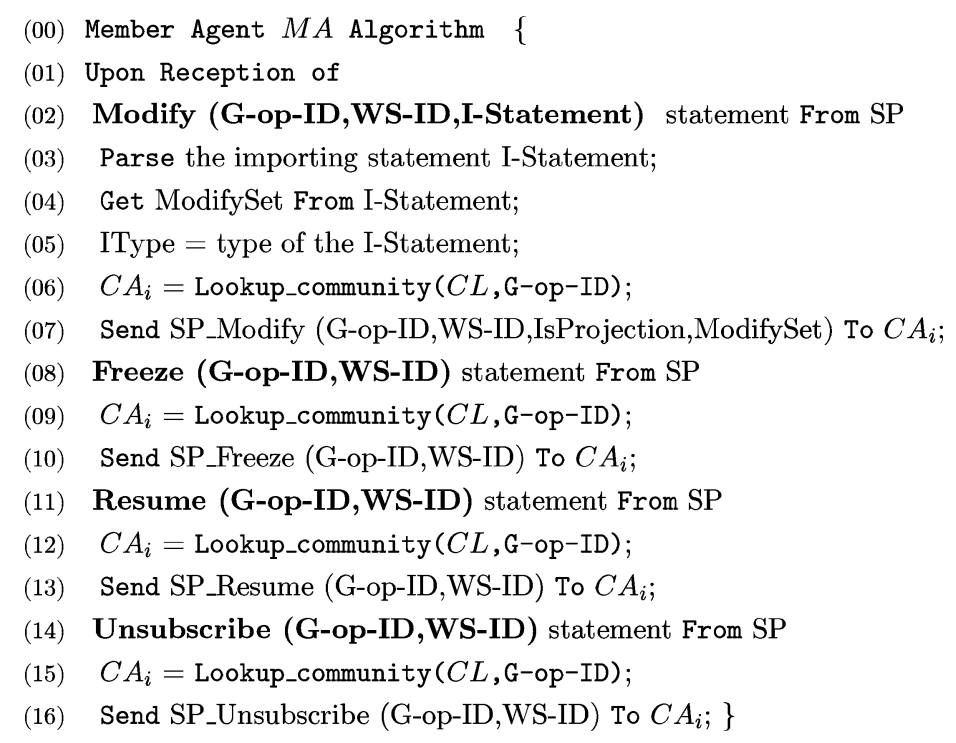

Figure 10. Changes issued by service providers: Member agent algorithm. 


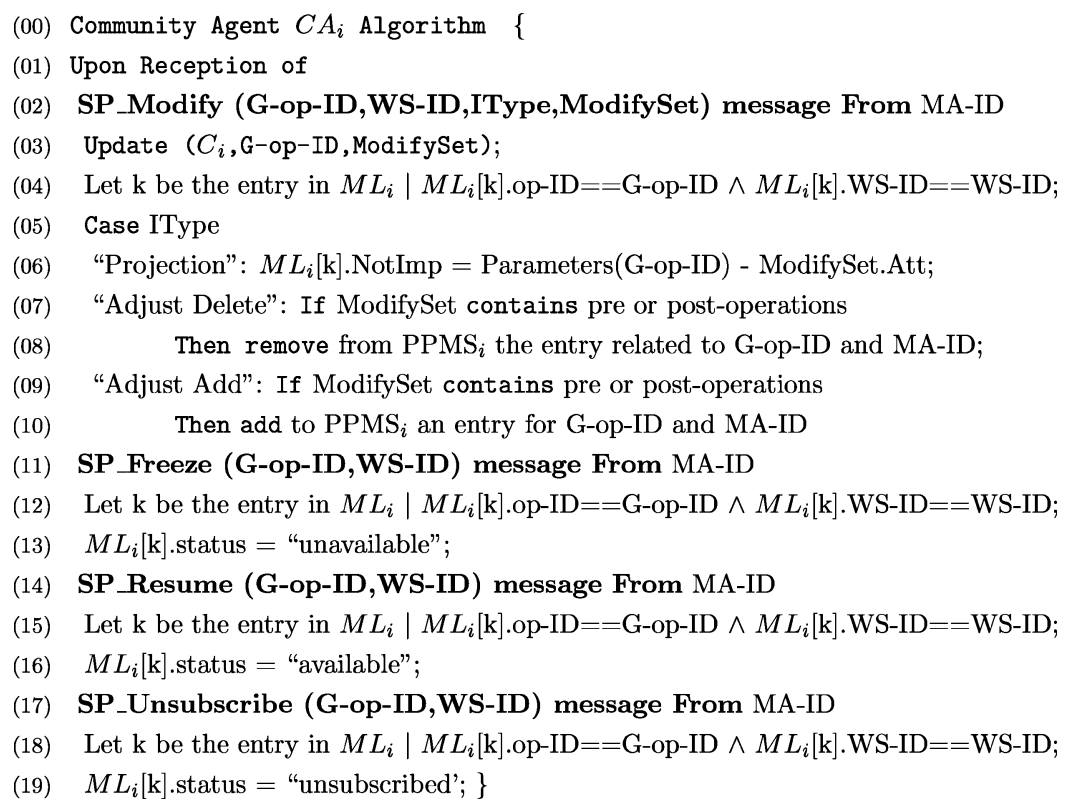

Figure 11. Changes issued by service providers: Community agent algorithm.

Rule $R_{1}$

Event CP-Modify(G-op-ID, $\mathrm{WS}_{j}$, ModifySet)

Condition Source $=C_{1} \wedge$ pre-operations $\in$ Modify.Att

Action $<$ notify the service provider to change its internal business logic $>$; send Resume (G-op-ID, $\left.W S_{j}\right)$ message to source

$R_{1}$ states that whenever the member receives a change notification issued by $C_{1}$ 's provider and if the change concerns the pre-operations attribute, the service provider should reflect the change by modifying its internal business logic. The member agent then sends a Resume $(G-$ $\left.o p-I D, W S_{j}\right)$ message to $C_{1}$ 's agent. The message is a confirmation that the member has locally reflected the changes done by $C P_{1}$. Figure 12 summarizes the change reaction algorithm executed by member agents.

\section{Implementation}

We provide a prototype implementation of the proposed approach using Web service standards such as SOAP and UDDI (figure 13). Users access the Semantic Web Service Manager through a graphical interface (implemented in HTML/Servlet). We identify two types of users: community providers and service providers. All users' requests are received by the request handler which forwards them either to the community builder or service registrar depending on their type. 


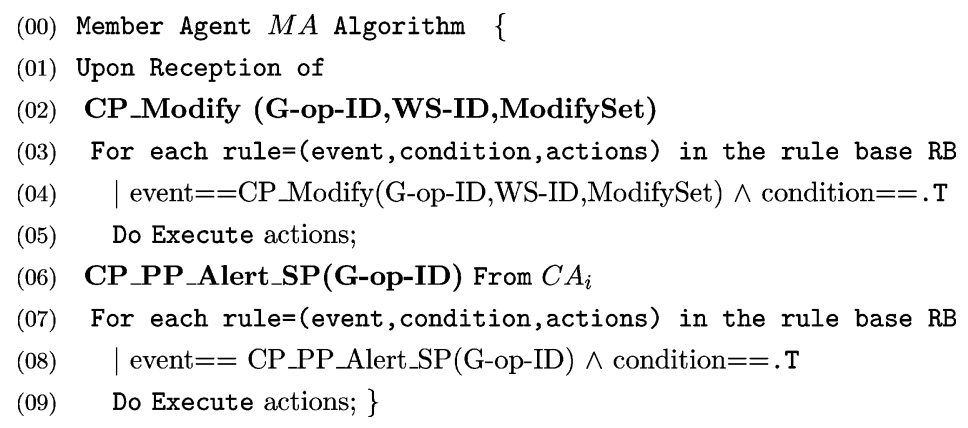

Figure 12. Reaction to changes issued by community providers: Member agent algorithm.

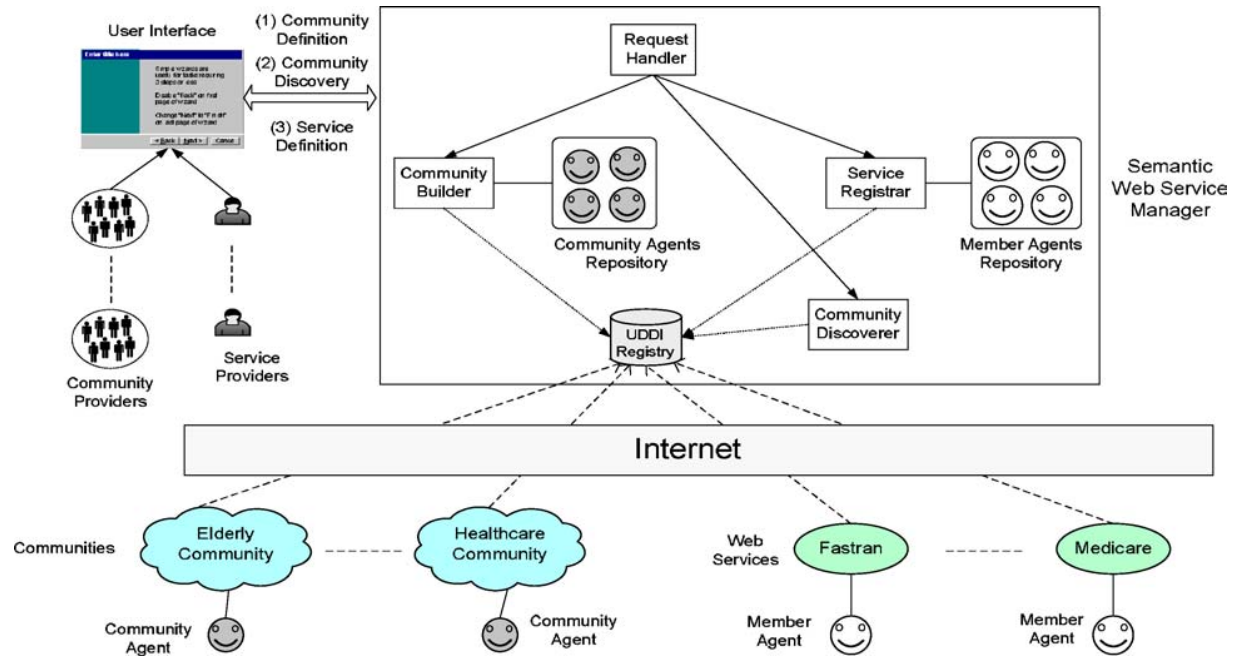

Figure 13. Architecture.

If the request is for creating a community (issued by community providers), it is handled by the community builder. Community providers define a community either by sending an XML document that instantiates the community ontology or by filling out a form via the graphical interface. In both cases, the community builder creates an entry for the community in the UDDI registry. We implement the UDDI with Systinet's WASP UDDI Standard 3.1. Cloudscape 4.0 database is used to create the registry for the UDDI. All communications are encapsulated in SOAP envelopes. Apache SOAP provides the tools necessary for deploying SOAP messaging. Every community is represented in the registry by a tModel [1]. We have designed specific tModels for communities. The community tModel contains a URL pointer to the description of the community. When defining a community, an agent called community agent (implemented using IBM Aglets) is downloaded from the community agents repository to the community provider's site. All subsequent access (e.g., modify a community) to the community are done through the community agent. 
The second type of requests concerns the discovery of communities. These requests are issued by service providers to inquire about communities of interest. They are received by the community discoverer which implements UDDI Inquiry Client using WASP UDDI API. Once a community is discovered, the service provider may submit a request for registration with this community. Such a request is forwarded to the service registrar which creates an entry for the Web service in the UDDI registry. The Web service is represented by a $t$ Model in the registry. We have also designed tModels for community members called member tModel. As a result of the registration process, a member agent (implemented using IBM Aglets) is downloaded to the service provider's site. The member agent is then responsible for handling all subsequent requests issued by the service provider. The deployment of community and member agents caters for a peer-to-peer and distributed architecture for managing communities and Web services.

\section{Related work}

Standardization efforts including UDDI (Universal Description, Discovery, and Integration) and WSDL (Web Services Description Language) are underway to support Web services [1]. The main impediment of current Web service standards is their limited support for semantics. A major effort towards enabling semantic Web services is DAML-S (DARPA Agent Markup Language) [10]. DAML-S defines a semantic markup of Web services based on ontologies. Several features distinguish the approach proposed in this paper from $D A M L-S$. First, $D A M L-S$ proposes an ontology for Web services not for community of Web services. Our approach provides means for the semantic description of Web services and their ontological organization into communities. Second, the static semantics in $D A M L-S$ mostly focuses on describing operations' features. We define a broader view of static semantics by describing semantics both at the operation and message levels. Third, DAML-S gives little support for the dynamic semantics of Web services. It does not allow the specification of pre-operations and post-operation which are particularlyimportant for enabling the automatic generation of business processes. Additionally, the notion of behavior and business logic is not explicitly defined. Fourth, $D A M L$-Sproviders define their service operations from scratch. In our approach, providers inherit the functionalities of a community simply by registering their services with it. They may also personalizethat community to best fit their capabilities.

WSMF (Web Service Modeling Framework) combines the concepts of Web services and ontologies to cater for semantic Web enabled services [4]. WSMF is still in its early stage. The techniques for the semantic description of Web services are still ongoing. Furthermore, WSMF does not address the issue of organizing semantic Web services.

\section{Conclusion}

In this paper, we propose an ontological framework to cater for a meaningful organization and description of Web services. We introduce the concept of community to cluster Web services based on their domain of interest. We develop an ontology called community ontology 
that serves as a "template" for describing communities and Web services. The proposed framework lays the foundation for the automatic selection and composition of semantic Web services. We are currently defining a composability model that would automatically check whether Web services "can" be combined together. The model would compare different features of Web services including syntactic, semantic (static and dynamic), behavioral, and qualitative properties.

\section{Acknowledgment}

The second author's research is partly supported by the National Institutes of Health's NLM Grant 1-R03-LM008140-01.

\section{References}

1. G. Alonso, F. Casati, H. Kuno, and V. Machiraju, Web Services: Concepts, Architecture, and Applications, Springer Verlag, June 2003, ISBN: 3540440089.

2. T. Berners-Lee, J. Hendler, and O. Lassila, “The Semantic Web," Scientific American, May 2001.

3. C. Bussler, B2B Integration, Springer Verlag, May 2003, ISBN: 3540434879.

4. C. Bussler, D. Fensel, and A. Maedche, "A conceptual architecture for semantic Web enabled Web services, SIGMOD Record, vol. 31, no. 4, 2002.

5. C. Collet, T. Coupaye, and T. Svensen, "Naos: Efficient and modular reactive capabilities in an object-oriented database system," in VLDB Conf., Santiago, Chile, September 1994.

6. T.H. Cormen, C.E. Leiserson, R.L. Rivest, and C.Stein, Introduction to Algorithms, MIT Press, 2001.

7. D. Fensel, Ontologies: A Silver Bullet for Knowledge Management and Electronic Commerce, Springer Verlag, Sept. 2003, ISBN: 3540003029.

8. Object Management Group, Unified Modeling Language Specification. http://www.omg.org/technology/ documents/formal/uml.htm, 1999.

9. I. Horrocks, "DAML+OIL: A description logic for the Semantic Web," IEEE Data Engineering Bulletin, March 2002.

10. S.A. Mclraith, T.C. Son, and H. Zeng, "Semantic Web services," IEEE Intelligent Systems, March 2001.

11. C. Petrie and C. Bussler, "Service agents and virtual enterprises: A survey," IEEE Internet Computing, vol. 7, no. 4, 2003.

12. S. Ran, "A model for web services discovery with QoS," SIGecom Exchanges, vol. 4, no. 1, 2003.

13. S. Tsur, S. Abiteboul, R. Agrawal, U. Dayal, J. Klein, and G. Weikum, "Are Web services the next revolution in e-commerce? (Panel)," in VLDB Conference, Sep. 2001.

14. G. Weikum (ed.), Special Issue on Organizing and Discovering the Semantic Web, IEEE Data Engineering Bulletin, March 2002.

15. L. Zeng, B. Benatallah, M. Dumas, J. Kalagnanam, and Q.Z. Sheng, "Quality driven Web services composition,” in Twelfth International World Wide Web Conference, Budapest, Hungary, May 2003. 